\title{
Anterior Decompression and Fusion with Japanese Clog-Shaped Bone Graft for Adjacent Multi-Level Cervical Disc Disease
}

\author{
Tadashi Kojima, Shiro Waga, Fumiaki Nakamura, \\ Masaki Mizuno, Minoru Kuroki
}

Department of Neurosurgery, Mie University School of Medicine, Tsu, Japan

\begin{abstract}
Between January 1987 and December 1996, we have performed anterior decompression and interbody fusion with a "Japanese clog" shaped bone graft in 21 patients with cervical myelopathy and/or radiculopathy caused by adjacent multilevel cervical disc disease. There were 17 men and 4 women, the mean age was 51 years, ranging from 25 to 71 years. Their affected levels consisted of C4/5 and C5/6 levels in 11 patients, $\mathrm{C} 5 / 6$ and C6/7 levels in 9 patients, and C4/5, C5/6 and $\mathrm{C} 6 / 7$ levels in one patient.

Surgery: After exposure of affected levels as usual fashion, the anterior half of the vertebral body between the two adjacent affected intervertebral discs is drilled out using a high speed drill. The upper part and lower part of the remaining vertebral body also are drilled out for $3 \mathrm{~mm}$ of their length. The transverse width of the drilled body is about 15 to $18 \mathrm{~mm}$. Then discectomy, osteophytectomy and resection of the posterior longitudinal ligament as well as removal of herniated disc materials are carried out at the level of the adjacent affected disc spaces. After sufficient decompression of the neural tissue, a trough is created in the vertebral bodies above and below to allow the bone graft to be wedged in. In Japan no bone bank is available, so a bone graft from the iliac crest is shaped in the form of a Japanese clog, and the cranial end inserted first. The caudal end is gently tamped into place. In all out one of our 21 patients, marked improvement of spondylotic myelopathy was gained.

In conclusion, the benefit of this procedure includes a wide working space, sure and safe manipulation of microinstruments, and safe insertion and complete fusion of the bone graft.
\end{abstract}

Key word: cervical disc disease, multiple anterior decompression, levels, Japanese clog-shaped bone graft

Spinal Surgery $12(2): 153 \sim 159,1998$

(Received : December 25, 1997, Accepted : February 23, 1998)

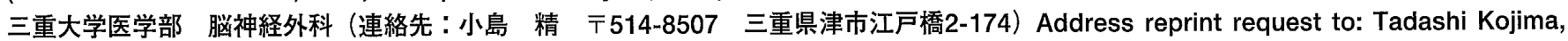
M.D., Department of Neurosurgery, Mie University School of Medicine, 2-174, Edobashi, Tsu, Mie, 514-8507 , Japan 


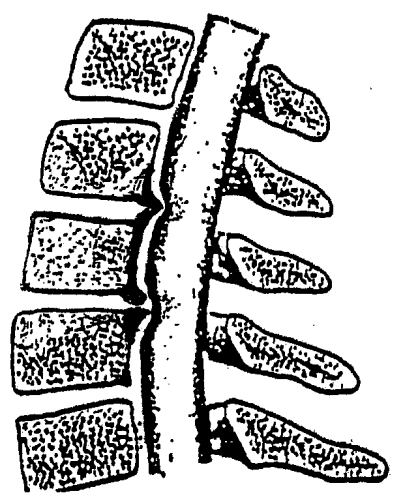

1A

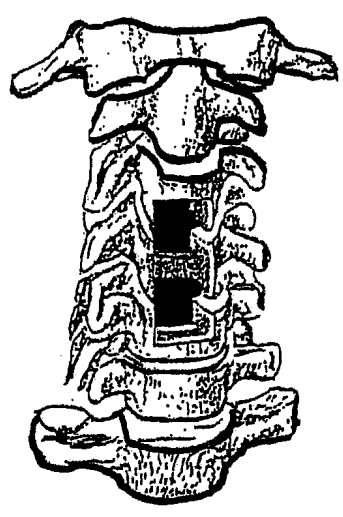

$1 B$

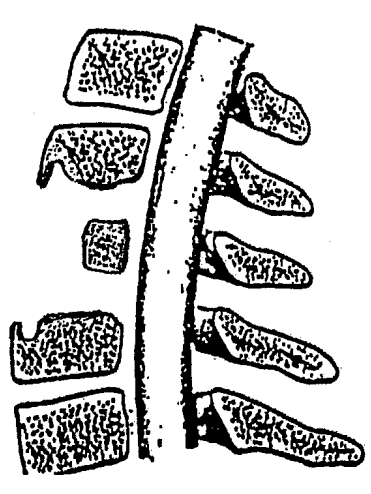

$1 \mathrm{C}$

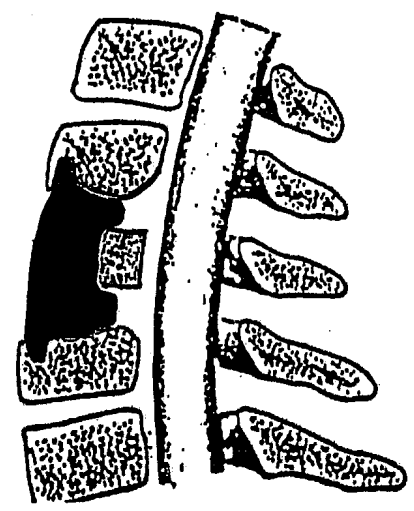

1D

Fig. 1: Drawings depicting adjacent two levels lesions (1 A), partial corpectomy of the vertebral body between adjancet affected disc spaces, and sufficient decompression of the two lesions (1 B), and interbody fusion with Japanese clog- shaped bone graft (1 C). (With permission from the Spinal Surgery)

\section{INTRODUCTION}

Multi-level cervical disc disease is approached surgically from a variety of anterior and posterior routes. The choice of surgical procedures, including laminectomy, laminoplasty, anterior fusion, and anterior decompression and fusion, remains controversial $[1,2,3$, $7,8,9]$. Historically, the most common operation for the treatment of cervical spondylotic myelopathy has been laminectomy. Unfortunately, the posterior approach sometimes exhibits neurological deterioration due to increasing kyphotic deformity and instability after the procedure, in addition to anterior cord compression. We have performed anterior decompression and interbody fusion with a Japanese clog-shaped bone graft in 21 patients with cervical myelopathy caused by adjacent multi-level cervical disc disease. The advantages of and the indications for this procedure are discussed.

\section{CLINICAL MATERIALS AND METHODS}

\section{$<$ Patients >}

Between January 1987 and December 1996, 120 patients with cervical disc disease underwent an anterior approach procedure. Twenty-one of the 120 patients had cervical myelopathy, radiculopathy, and/or radiculomyelopaty caused by adjacent multilevel cervical disc disease. These 21 patients, (17 men and 4 women, mean age 51 years, ranging 25-71 years) were operated on using anterior decompression with a Japanese clog-shaped bone graft for all involved levels. A cervical spine radiographic series and axial computed tomographic (CT) scan after injection of contrast material into the lumbar subarachnoid space and/or magnetic resonance imaging (MRI) were obtained for each patient. Their affected levels consisted of C4/5 and C5/6 levels in 11 patients, $\mathrm{C} 5 / 6$ and $\mathrm{C} 6 / 7$ levels in 9 patients and $\mathrm{C} 4 / 5$, $\mathrm{C} 5 / 6$ and $\mathrm{C} 6 / 7$ levels in one patient. 


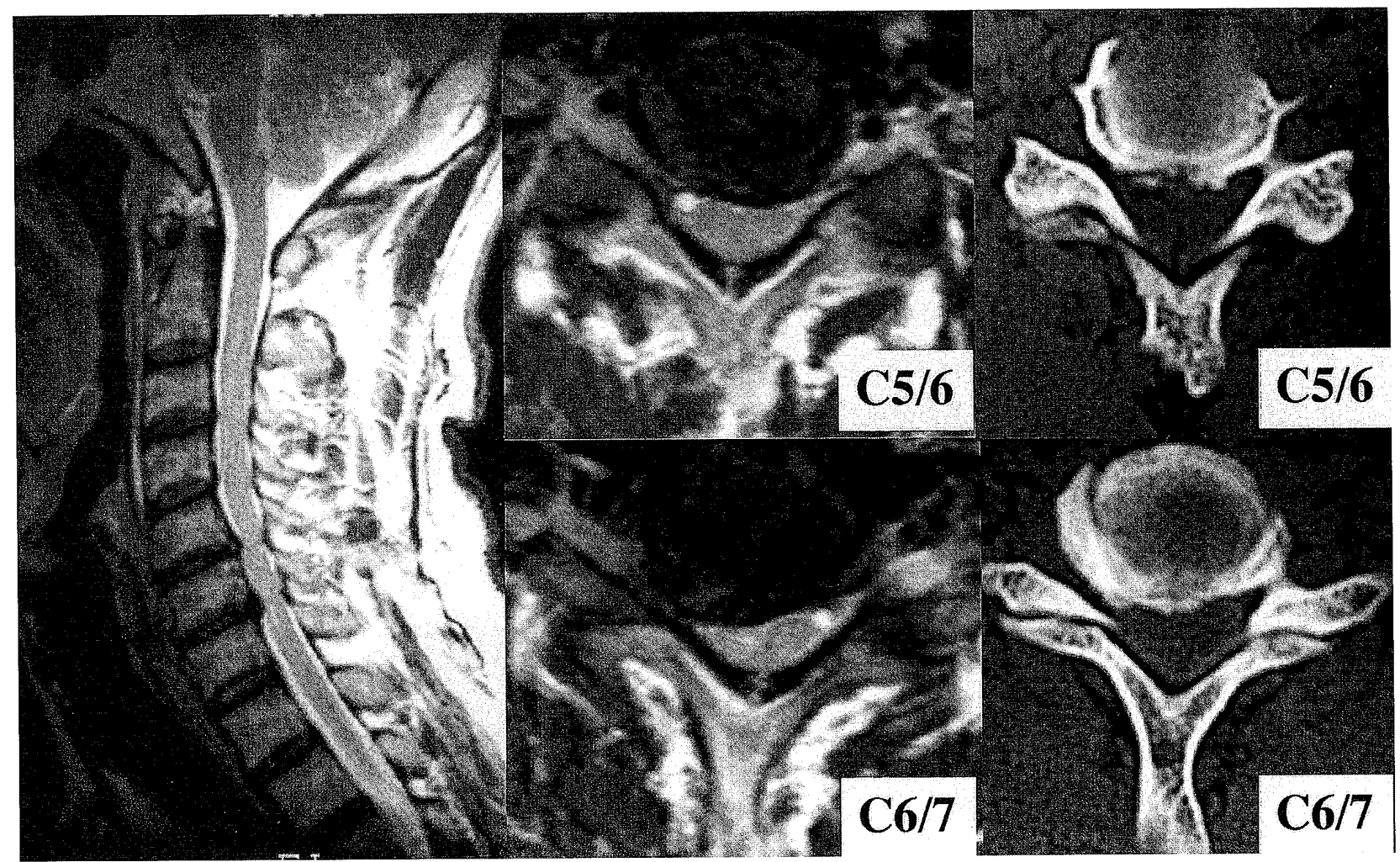

2C

2A

2B

Fig. 2: Illustrative case. Preoperative MRI showing the spinal cord compression at the levels of C5/6 and C6/7 (2 A, 2 B). Preoperative CT scans revealing osteophyte at the level of C5/6 (2 C) and disc herniation at the level of $\mathrm{C} 6 / 7(2 \mathrm{D})$.

\section{< Surgical Technique >}

After endotracheal intubation and the induction of general anesthesia, the patient is placed in the supine position. The patient's head is slightly extended and rests on a Mayfield padded horseshoe. A transverse cervical incision in an appropriate crease is then completed, followed by a routine transverse incision of the platysma muscle. Localization of the prevertebral compartment is then accomplished with blunt dissection initiated anterior to the border of the right sternocleidomastoid muscle. This muscle is mobilized laterally, along with the carotid sheath. The esophagus, trachea, and strap muscles are retracted medially. After the appropriate spinal level is confirmed by intraopertive X-ray films, the anterior longitudinal ligament and longus colli musculatures are dissected $20 \mathrm{~mm}$ in width. The anterior aspect of the cervical spine is identified. Using a high speed drill, the anterior half of the vertebral body between the two adjacent affected intervertebral discs is drilled out, and the upper and lower parts of the remaining vertebral body also are drilled out for $3 \mathrm{~mm}$ their length. The transverse width of the drilled body is about 15 to $18 \mathrm{~mm}$. Discectomy, osteophytectomy and resection of the posterior longitudinal ligament as well as removal of herniated disc materials are carried out safely and accurately at the levels of the adjacent affected disc spaces with a wide view under the operative microscope. As a result, the central onethird of the vertebral body remains. After sufficient decompression of the neural tissue, a trough is created 


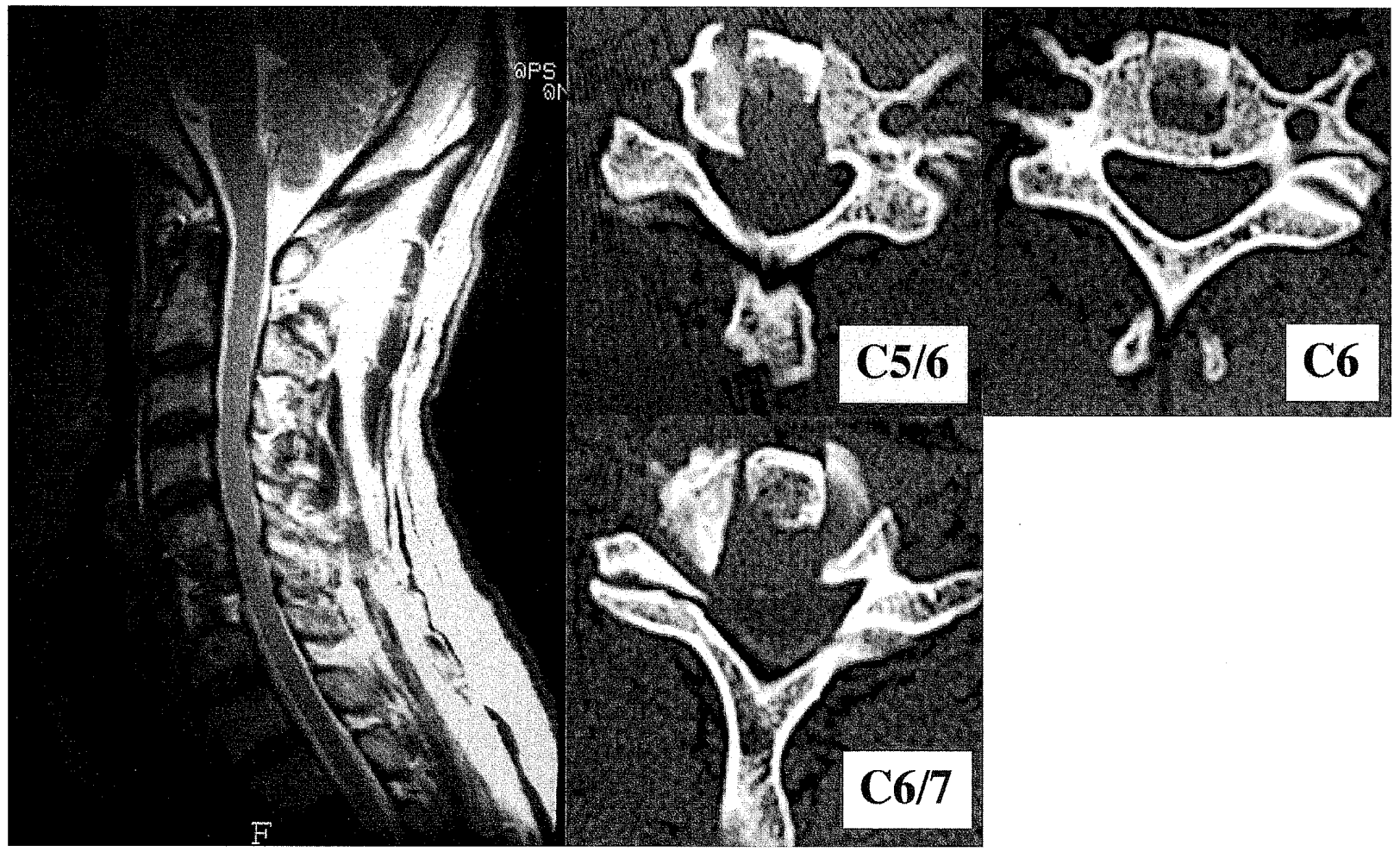

Fig. 3: Illustrative case. Postoperative MRI demonstrating sufficient decompression at the levels of C5/6 and C6/7 (3 A). Postoperative CT scans demonstrating sufficient decompression at the levels of C5/6 (3 B) and $\mathrm{C} 6 / 7$ (3 C), and partly remaining $\mathrm{C} 6$ vertebral body (3 D).

in the vertebral bodies above and below to allow the bone graft to be wedged in. Then a bone graft from the iliac crest is shaped in the form of a Japanese clog, the cranial end of the graft is inserted first, and the caudal end is gently tamped into place(Fig. 1). Postoperatively, a cervical collar is applied for 8 weeks.

\section{RESULTS}

Follow-up periods varied from 1 to 9.5 years, with a mean of 59 months. Fusion of the bone graft and alignment of the cervical spine were confirmed radiographically in all patients by plain cervical Xrays and CT scans. Decompression of the the spinal cord was confirmed postoperatively by MRI. The state of the bone graft was checked by follow-up Xray films 3,6 , and 12 months after surgery. Nonunion or absorption of the bone graft has not observed in all cases, and good alignment was gained. In all but one of 21 patients, a marked improvement of preoperative neurological symptoms was gained. Clinical results were evaluated according to the "Neurosurgical cervical spine scale" (NCSS) [6]. The NCSS quantifies neurological impairment by evaluating lower extremity motor function (5 points), upper extremity motor function ( 5 points), and sensory function and/or pain (4 points). Therefore, a perfect NCSS score is 14 points. Recovery rate can 
be expressed by the following formula.

Recovery rate $(\%)=$ (postoperative score-preoperative score/14-preoperative score) x 100

Preoperative NCSS score averaged 9.1 points (ranging from 3 to 12). The postoperative score was 12 points (ranging from 9 to 14). Clinical recovery rate averaged $62.5 \%$. In one patient associated with cervical ossification of the posterior longitudinal ligament, postoperative improvement of the myelopathy was gained temporarily, but his myelopathy deteriorated 3 weeks after surgery, necessitating immediate corpectomy and removal of the ossification of the posterior longitudinal ligament.

\section{CASE REPORT}

A 50-year-old man was admitted to our hospital because of gait disturbance, and clumsiness and dysesthesia of hands for 3 months. A neurological examination on admission showed tetraparesis, hypesthesia, hyperactive deep tendon reflexes of the four extremities and muscle atrophy of the right hand. A spinal CT scan demonstrated posterior spurs and disc herniation at the levels of $\mathrm{C} 5 / 6$ and $\mathrm{C} 6 / 7$. Sagittal MRI showed the spinal cord compression at the levels of $\mathrm{C} 5 / 6$ and $\mathrm{C} 6 / 7$, and axial MRI revealed compression of the spinal cord on the left side at the level of $\mathrm{C} 5 / 6$ and on the right side at the level of $\mathrm{C} 6 / 7$ (Fig. 2). After dissection of the anterior longitudinal ligament and longus colli musculature, $20 \mathrm{~mm}$ in width, the anterior half of the vertebral body of C6 was drilled out $18 \mathrm{~mm}$ in width, then the cranial and caudal parts of the residual vertebral body of C6 were drilled out about $3 \mathrm{~mm}$ in length. Microdiscectomies and osteophytectomies at the levels of C5/6 and C6/7 could be done accurately with a wide working space. Following excision of the posterior longitudinal ligament, good pulsation of the dural theca was gained. After sufficient decompression of the neural tissue, a Japanese-clog shaped bone graft from the iliac crest was inserted safely(Fig. 3). Postoperatively, a cervical collar was applied for 8 weeks. The patient's neurological condition improved soon after surgery. Postoperative cervical X-ray demonstrated good alignment. He was discharged without any neurological deficit.

\section{DISCUSSION}

Surgical management is indicated for patients with progressive or persistent neurological deficit and intractable pain and neurodiagnostic imaging showing compression of the cervical spinal cord and/or roots [1-5, 7-9]. A number of operative procedures have been utilized to relieve compression of the cervical spinal cord and nerve roots caused by disc disease. The ideal approach is one which most directly and safely allows for the removal of offending compressive pathology with complete decompression of the cervical spinal cord and/or nerve roots, such as anterior approach. In most instances, either the anterior or posterior approach can be used with satisfactory results. However, for the vast majority of patients with cervical myelopathy and/or radiculopathy, the anterior approach has proven to be the most efficacious operative option, because herniation of the ventrally-located disc typically results in ventrolateral cord/root compression. Also a number of operative procedures using an anterior approach have been reported, including anterior approach with bone graft, anterior approach without bone graft, and anterior vertebrectomy with interbody fusion. Isu et al. [5] proposed that anterior cervical fusion utilizing bone graft obtained from cervical vertebral bodies is useful in the surgical treatment of cervical disc disease. According to them, in the small operative field of the Robinson-Smith technique carries a risk of damage to the spinal cord or nerve root when complete removal of the osteophyte is attempted. Their procedure provides more extensive exposure of the osteophyte and the herniated disc, which can be safely and easily removed without damage to the spinal cord. Previously we reported an anterior vertebractomy and interbody fusion for multi-level cervical spondylosis [7]. Recently, in patients with adjacent multi-level cervical disc 
disease, we have surgically removed vertebral bodies between the affected adjacent discs subtotally, as only the central third of the body remained following excision of the posterior margins of the upper and lower vertebral end-plates together with the osteophytes and herniated discs, from a wide operative field. A single bone graft from the iliac crest was shaped in the form of a Japanese clog, and then inserted gently with tapping. Tapping of the bone graft is very safe and easy because the posterior part of the vertebral body between two affected intervertebral spaces remains as a bar of bone with a vertical width of $5 \mathrm{~mm}$. Hirabayashi and Uzawa [4] reported as follows: When a vertebral body is removed subtotally in the process of a multilevel cervical canal decompression, only the central third of it remains following excision of the posterior margins of the upper and lower vertebral end-plate. Two threads pass beneath the remenant vertebral body and tie firmly to a strut graft to prevent extrusion of the bone graft.

The number of cases in this series is limited and the follow-up period is short. In conclusion, the benefit of this procedure includes wide working space, accurate and safe manipulation of microinstruments, and safe insertion of the bone graft.

\section{REFERENCES}

1. Blades DA, Cooper PR : Management of cervical disk herniation: Anterior surgical approaches. In: Menezes AH, Sonntag VK editors. Principles of the Spinal Surgery, Vol 1. New York: McGrawHill, 1996: 517-529.

2. Hardley MN, Sonntag VK : Cervical disk herniations: The anterior operative approach without interbody fusion. In: Menezes AH, Sonntag VK editors. Principles of Spnial Surgery, Vol 1. New York: McGraw-Hill, 1996: 531-538.

3. Hanai K, Fujiyoshi F, Kamei K : Subtotal vertebrectomy and spinal fusion for cervical spondylotic myelopathy. Spine 1986;11: 310-315.

4. Hirabayashi $\mathrm{K}$, Uzawa $\mathrm{M}$ : Multilevel anterior cervical interbody fusion: a new method of subcortical binding to prevent graft dislocation. Neuro-Orthopedics 1995;17/18: 21-28.

5. Isu T, Kamada K, Kobayashi N, Mabuchi S : The surgical technique of anterior cervical fusion using bone grafts obtained form cervical vertebral bodies. J Neurosurg 1994;80: 16-19.

6. Kadoya S: Grading and scoring system for neurological function in degenerative cervical spine disease. - Neurosurgical cervical spine scale -Neurol Med Chir(Tokyo) 32: 40-41,1992.

7. Kojima T, Waga S, Kubo Y, Kanamaru K, Shimosaka S, Shimizu T : Anterior cervical vertebrectomy and interbody fusion for multi-level spondylosis and ossification of the posterior longitudinal ligament. Neurosurgery 1989;24: 864-872.

8. Saunders RL, Bernini PM, Shirreffs TG, Reeves AG : Central corpectomy for cervical spondylotic myelopathy : a consecutive series with long-term follow-up evaluation. J Neurosurg 1991;74: 163170.

9. Whitecloud TS III : Anterior surgery for cervical spondylotic myelopathy: Smith-Robinson, Cloward and vertebrectomy. Spine 1988;13: 861863. 


\section{Reviewer's comment : \\ Tomokazu Goya \\ Department of Neurosurgery, Miyazaki Medical College \\ Miyazaki, Japan}

The authors provide a variable alternative of anterior approach to multi-levels cervical disc disease. I enjoyed the author's interesting operative procedure for treatment of myeloradiculopathy due to cervical spondylosis and disc hernia.

This somewhat complicated procedure has benefits of surgery, such as wide operative field, prevention of posterior displacement of the graft bone and accuracy of bony fusion.

This method is useful to treat two disc levels spondylosis and disc hernia. And it may be probably inappropriate to treat three disc levels, and also unsuitable for removal of cervical OPLL through anterior approach, as described in the result. This method should be confined to cervical spondylosis and disc hernia in two disc levels.

\section{Reviewer's comment : \\ Kazutoshi Hida \\ Department of Neurosurgery, University of Hokkaido School of Medicine Sapporo, Japan}

This article presents an interesting method of the anterior fusion named by the authors as "Japanese clogshaped bone graft". The authors demonstrated their experience of this surgical technique on cervical spondylosis and cervical OPLL. They described surgical procedures in detail. The results presented seem satisfactory.

There has been a controversy what is the best way to treat patients with cervical spondylosis. The advantage of this procedure seems to secure a wide operative view and to prevent extrusion of the grafted bone. However, this method sacrifice the vertebral body which is unnecessarily taken out. Another criticism is its nomenclature "Japanese clog style", which is rather difficult to be understood by most of neurosurgeon in the other countries. 